\title{
What triggers star formation in galaxies?
}

\author{
Bruce G. Elmegreen \\ IBM Research Division, T.J. Watson Research Center \\ 1101 Kitchawan Road, Yorktown Heights, NY 10598 USA \\ email: bge@us.ibm.com
}

\begin{abstract}
Processes that promote the formation of dense cold clouds in the interstellar media of galaxies are reviewed. Those that involve background stellar mass include two-fluid instabilities, spiral density wave shocking, and bar accretion. Young stellar pressures trigger gas accumulation on the periphery of cleared cavities, which often take the form of rings by the time new stars form. Stellar pressures also trigger star formation in bright-rim structures, directly squeezing the pre-existing clumps in nearby clouds and clearing out the lower density gas between them. Observations of these processes are common. How they fit into the empirical star formation laws, which relate the star formation rate primarily to the gas density, is unclear. Most likely, star formation follows directly from the formation of cold dense gas, whatever the origin of that gas. If the average pressure from the weight of the gas layer is large enough to produce a high molecular fraction in the ambient medium, then star formation should follow from a variety of processes that combine and lose their distinctive origins. Pressurized triggering might have more influence on the star formation rate in regions with low average molecular fraction. This implies, for example, that the arm/interarm ratio of star formation efficiency should be higher in the outer regions of galaxies than in the main disks.
\end{abstract}

Keywords. stars: formation, ISM: bubbles, galaxies: spiral

\section{The Galactic Scale}

When we observe a star-forming region we sometimes wonder how it got there, or why stars formed there, or anywhere for that matter. The average gas density in most galaxies is very low, close to the tidal limit of $\rho_{\text {tidal }}=-1.5 \Omega R(d \Omega / d R) /(\pi G) \sim 1 \mathrm{~cm}^{-3}$ for galactic rotation rate $\Omega$ and radius $R$. This is also about the gas surface density limit where Toomre $Q=\sigma_{\text {gas }} \kappa /\left(\pi G \Sigma_{\text {gas }}\right) \sim 1$ for velocity dispersion $\sigma_{\text {gas }}$, epicyclic frequency $\kappa$, and mass column density $\Sigma_{\text {gas }}$. Stellar explosions produce denser gas in supernova remnants, but this gas is usually too tenuous to be strongly self-gravitating, as in the Veil Nebula, and it is also too short-lived in that state for self-gravity to operate (Desai et al. 2010). The two-phase instability makes cool gas, but this operates only between temperatures of $\sim 10,000 \mathrm{~K}$ and $\sim 100 \mathrm{~K}$, which is not cold enough to make stars. Star formation requires a thermal Jeans mass close to a solar mass. The thermal Jeans mass is $M_{\mathrm{J}, \mathrm{th}}=\rho k_{\mathrm{J}}^{-3} \sim 7(T / 10 \mathrm{~K})^{1.5} n^{-0.5} M_{\odot}$ for thermal Jeans wavenumber $k_{\mathrm{J}}=(4 \pi G \rho)^{1 / 2} / c$ and isothermal sound speed $c=(k T / \mu)^{1 / 2}$ with mean weight $\mu$ of atomic gas, and density $n=\rho / \mu$. For the range of temperatures expected from the thermal instability and for the average density, $M_{\mathrm{J}, \mathrm{th}} \sim 200 M_{\odot}$, which is too large for a star. Star formation requires gas that is both cold and dense to bring $M_{\mathrm{J}, \mathrm{th}}$ down. Processes that do this may be thought of as star-formation triggers.

A fair question is whether the ISM can make cold and dense gas on its own, without non-ISM processes, such as stellar density waves, galactic shear, superbubbles, etc.. If there were no stars to compress the gas or supplement its gravitational self-attraction 
with additional mass, would new stars form? The answer is probably yes because galaxies once had no stars, and the gas formed stars anyway. Still, galaxy collisions in the young universe could have triggered the star formation by forcing the gas to be dense in the shocked overlap region and in a central concentration that formed after angular momentum loss.

During the last few years, observations of very young galaxies seem to suggest that they can form giant clumps of star formation on their own, even with no observable underlying disk of older stars and no evidence for an interaction with another galaxy (Elmegreen et al. 2009ab, Genzel et al. 2011). The only possible process for this seems to be a gravitational instability in the whole gas disk, with a resulting clump mass comparable to the turbulent Jeans mass, $M_{\mathrm{J}, \mathrm{turb}}=\Sigma_{\text {gas }} k_{\mathrm{J}, 2 \mathrm{D}}^{-2}$ for $k_{\mathrm{J}, 2 \mathrm{D}}=\pi G \Sigma_{\text {gas }} / \sigma_{\text {gas }}^{2}$. The clumps in young galaxies are large relative to the galaxy radii because the turbulent speeds are large relative to the rotation speeds $v_{\text {rot }}$ (Davies et al. 2011). This follows from $R k_{\mathrm{J}, 2 \mathrm{D}} \sim G M /\left(R \sigma_{\text {gas }}^{2}\right) \sim\left(v_{\text {rot }} / \sigma_{\text {gas }}\right)^{2}$.

This process of gravitational collapse of the ISM is the most fundamental of triggering mechanisms. To carry the initial collapse all the way to stars in a young ISM may have also relied on the pervasive presence of CO molecules (Daddi et al. 2010, Tacconi et al. 2010), which allows for cooling to $T<100 \mathrm{~K}$, and on the extremely high $\Sigma_{\text {gas }}$, which lowers $M_{\mathrm{J}, \mathrm{th}}$ by increasing the average midplane density. If we write the midplane pressure in a puregas disk as $P=0.5 \pi G \Sigma_{\text {gas }}^{2}$ and the thermal Jeans mass as $M_{\mathrm{J}, \mathrm{th}}=c^{4}(4 \pi G)^{-1.5} P^{-0.5}$, then

$$
M_{\mathrm{J}, \mathrm{th}}=3.8(T / 100 \mathrm{~K})^{2}\left(\Sigma_{\mathrm{gas}} / 100 M_{\odot} \mathrm{pc}^{-2}\right)^{-1} M_{\odot}
$$

from which it can be seen that the observed ISM column densities in the clumps of young galaxies, $\Sigma_{\text {gas }}>100 M_{\odot} \mathrm{pc}^{-2}$ (Tacconi et al. 2010), raise the pressure so much that even temperatures of $100 \mathrm{~K}$ may be low enough for star formation. Collapse inside these clouds raises the pressure more because of the higher local column density in the resulting core, and it lowers the temperature more because of opacity.

When stars are present, gravitational instability in the ISM is augmented by stellar gravity. This happens most commonly in three ways: by a two-fluid swing-amplified instability (Toomre 1981), in the shocks of global spiral density waves (Roberts 1969), and in the dense central regions formed by gas accretion in a bar potential (Matsuda \& Nelson 1977).

The first of these involves the simultaneous collapse of gas and stars, which produces a moderately dense clump of both components on a timescale $\left(G \rho_{\text {total }}\right)^{-1 / 2} \sim \kappa^{-1}$ for $Q \sim$ 1. The stellar part of this collapse bounces as the stars move away with enhanced energy in epicycles. Nearby stars form a spiral wake (Julian \& Toomre 1966). The dissipative gas remains as a dense clump at the center of gravity of the instability, with a gas filament also forming in the wake. This is the two-fluid swing-amplified instability (Jog \& Solomon 1984, Rafikov 2001, Romeo \& Wiegert 2011, Elmegreen 2011). It appears to be common in galaxies, taking the form of multiple spiral arms that are long and irregular in the outer parts. Usually these arms are more regular in the inner regions, where multiplearms often merge into symmetric two-arm structures midway in the disk (Elmegreen \& Elmegreen 1995).

The second way in which stellar gravity augments the condensation of gas into starforming clumps is through stellar density waves, which arise in the gas+star mixture as a result of perturbations from the outer disk, companion galaxies, or bars, and which may last for several rotations (Lin \& Shu 1964) before moving to the center (Toomre 1969). These waves move through the gas and stars (unlike the swing amplifier instability which follows the gas +star mixture for a time $\kappa^{-1}$ ) and they shock the gas as it passes 


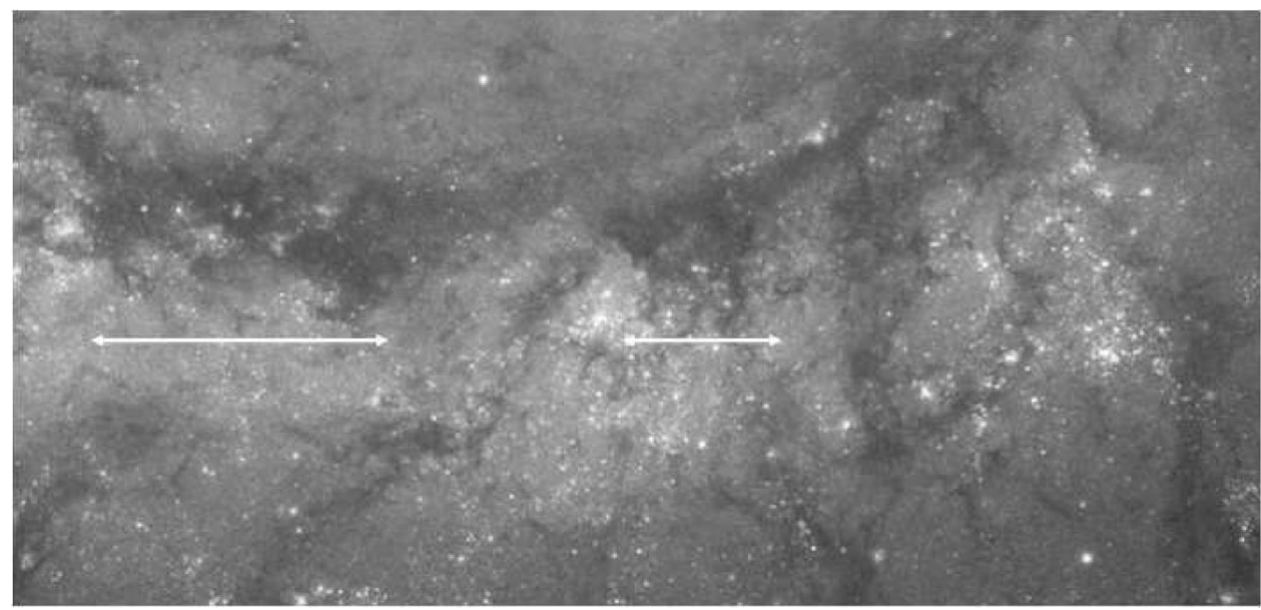

Figure 1. HST image of the southern arm in M51, showing large dark clouds, star formation in the clouds, and young stars downstream with shells and rings around them. The gas flows from the top left to the bottom right in the figure. The large dark clouds contain around $10^{7} M_{\odot}$ of gas. The shells and rings appear to contain new star formation along the periphery.

through. The shock appears as a thin dust lane. If the shocked gas is dense enough, it can become gravitationally unstable and form giant clumps and star complexes. It can also form stars by cooling interarm gas and squeezing interarm clouds. Most star formation in the Milky Way is in giant molecular clouds (GMCs) that are parts of $10^{7} M_{\odot} \mathrm{HI}+\mathrm{CO}$ complexes in spiral arms (Grabelsky et al. 1987). Because shear is low in spiral density wave arms, the process of gas collapse can be augmented by magnetic tensional forces, which remove angular momentum (Elmegreen 1987, Kim et al. 2002).

Some galaxies have a stellar component that is either too warm or too rapidly rotating for its column density to form strong spiral arms, i.e., $Q_{\text {stars }}>>1$; then the gas + star mixture is not particularly unstable. The gas is dissipative, however, and can still collapse on its own, or with only a small contribution from underlying stars. The result is a network of thin and short gaseous arms that form stars, giving a flocculent appearance. These arms should be co-moving with the material close to the site of the initial collapse, although they can be wave-like far from this site, in the spiral wake.

Figure 1 shows a piece of the galaxy M51 from an image taken with the Hubble Space Telescope. There is a spiral density wave with two large concentrations of gas in the dust lane, $10^{7} M_{\odot}$ each, and star formation in each concentration. When there are several of these concentrations along part of an arm, they look like "beads on a string". Efremov (2010) studied the relation between such regular patterns and the magnetic field. For several magnitudes of extinction, and for a thickness through the plane of $\sim 100 \mathrm{pc}$, the average density in the dustlane is $\sim 10 \mathrm{~cm}^{-3}$ (D. Elmegreen 1980) and so the average dynamical time is $\sim(G \rho)^{-1 / 2} \sim 30$ Myr. This average density is about what is expected for a density wave shock where the average incident density is the intercloud value $(\sim 0.3$ $\mathrm{cm}^{-3}$ ) and the ratio of the shock velocity to the internal turbulent speed is $\sim 5$. The average dynamical time is too long for gravitational collapse to develop much while the gas is in the dust lane, but the interarm gas is clumpy and these clumps feel a pressure increase when they enter the arm, causing them to collapse. Kim \& Ostriker (2002) showed the process of dustlane collapse starting with a smooth ISM. It took several rotations to build up enough density structure for a self-gravitating cloud to form in a spiral arm. 


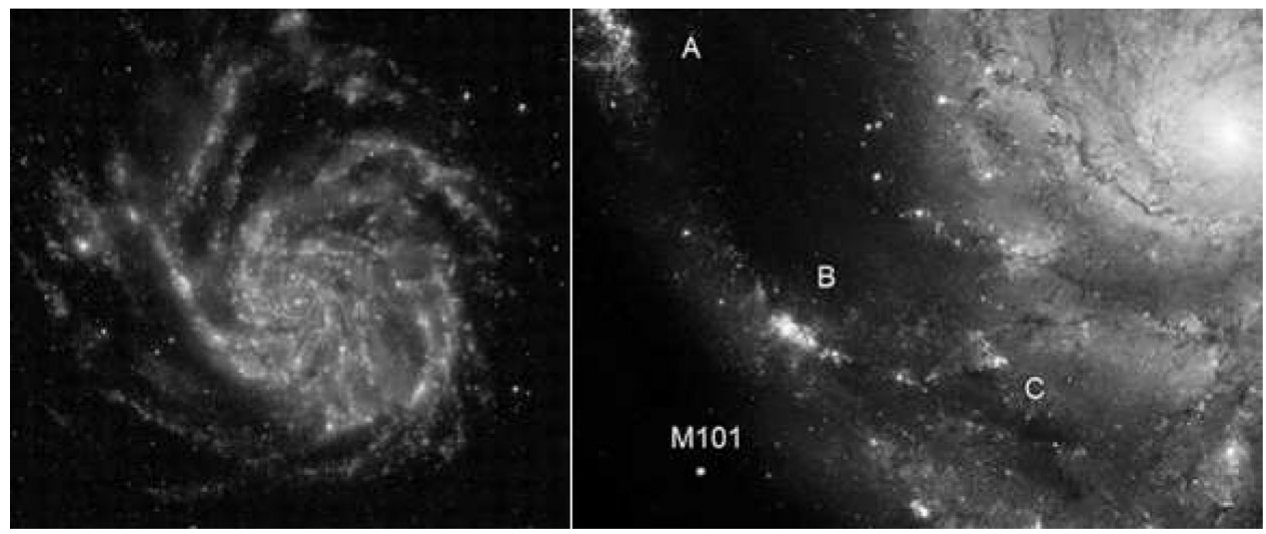

Figure 2. (Left) GALEX image in the uv of M101 from Gil de Paz et al. (2007). (Right) HST image of the western part of M101, showing two giant star complexes (A and B) and a dark cloud without much star formation yet (C). Young stars are centered in the spiral arms of M101, suggesting these arms are material patterns made from local gravitational collapse.

The structure of gas in the interarms is a remnant of the structure it had leaving the previous arm, which can also be seen in Figure 1. As the ISM moves through the arm, star formation pressures from HII regions, winds, and multiple supernovae make superbubbles and super-rings. The figure shows many rings of dust. The small rings can be three-dimensional shells seen with enhanced absorption along the lines of sight through the edges, but the large rings are much bigger than the ISM scale height and have to be two-dimensional. All of these structures seem to contain active star formation in the peripheral dense gas, in addition to OB associations and star complexes inside the cleared regions. Some of the active star formation is lingering in the dense clouds, which means that it follows the dense gas as it moves. Other active star formation on the periphery is probably triggered by the high pressures that made the cavities. This is a second type of triggering for star formation: sequential triggering by previous generations of stars. We review this in the next section.

Figures 2 and 3 show a multiple-arm galaxy, M101, and a flocculent galaxy, NGC 5055. The spiral structure in M101 is more irregular than in the grand design galaxy M51, but there are still long spiral arms and each arm has several regularly-spaced giant star complexes in it, in various stages of collapse. The right-hand side of Figure 2 highlights the western arm in M101, suggesting that the giant dust cloud toward the bottom is in a younger stage than the two other star complexes toward the west. Unlike in M51, the star formation in M101 seems to be centered on the arms with no rings offset to one side. This is expected when the whole arm is the result of a gravitational instability, because it all twists around with local shear and has little relative motion between the pattern speed and the gas. Figure 3 shows numerous long and thin spiral arms with more of the "beads on a string" pattern. These arms are so thin and irregular that they should be mostly gas. They shear around, stretch out, and disperse over time, most likely forming stars by gravitational collapse along their length. Rings and shells are not visible in this image. It would be interesting to observe further whether flocculent galaxies produce the same type of ring pattern as observed down stream from the spiral arms in M51.

The third common way in which stars in a galaxy promote gravitational collapse in the gas is through bar-driven inflows. Bars exert strong negative torques inside their corotation radii, which are typically at 1.2 to 1.4 bar radii. Inside this, the gas hits the bar from behind and shocks, forming a dustlane (Athanassoula 1992). The shock strips 


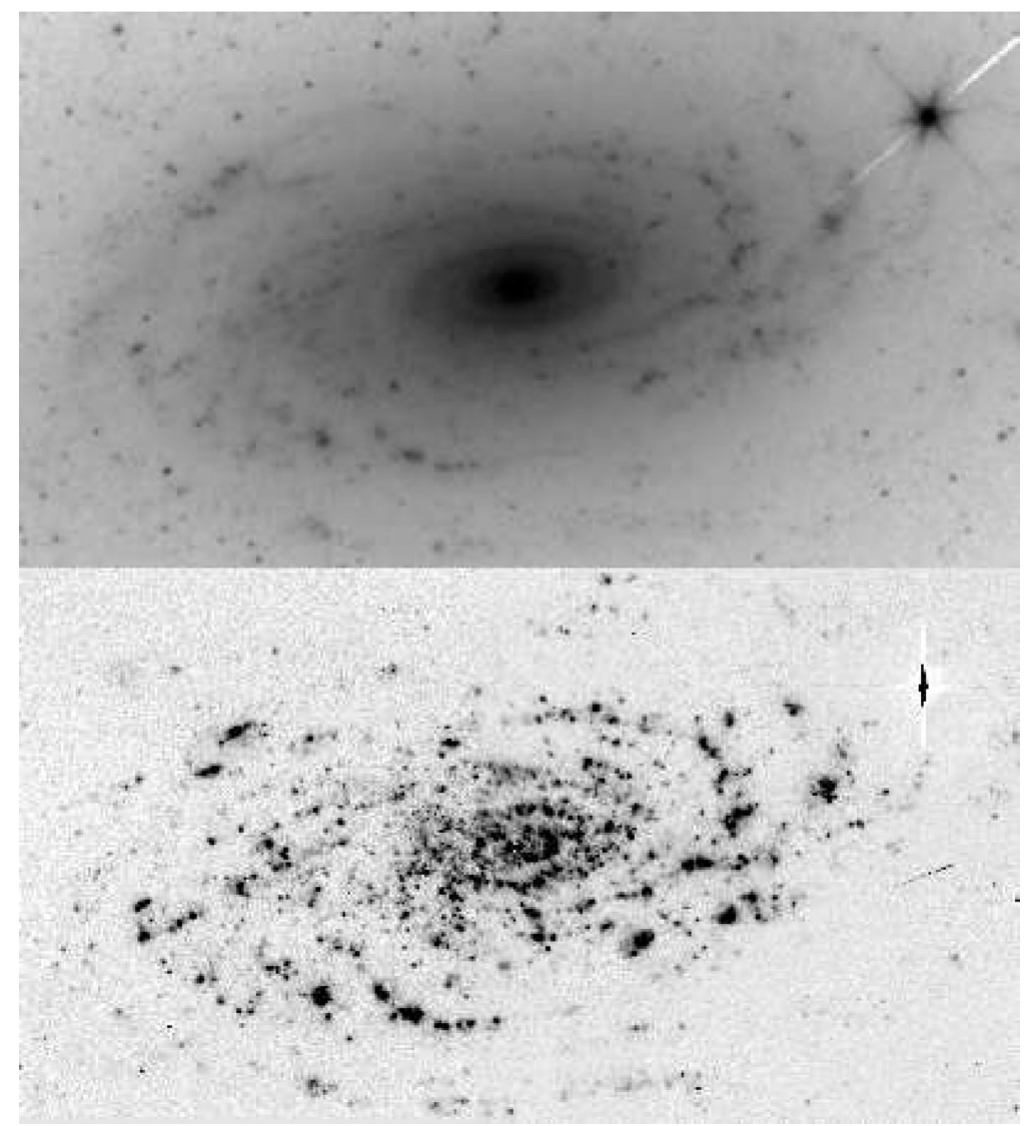

Figure 3. (top) Spitzer 3.6 $\mu$ image from Elmegreen et al. (2011) showing weak and irregular underlying stellar waves. (bottom) $\mathrm{H} \alpha$ image from Dale et al. (2009) showing numerous beads on a string of star formation.

angular momentum and orbital energy from the gas, which then falls along the bar to the region of the inner Lindblad resonance (ILR). After sufficient accumulation, the gas becomes unstable and form stars. Often it does this in a ring or tight two-arm spiral. There may be a second, smaller bar inside the ILR which affects the orbits there and brings the gas in further.

Figure 4 shows how the gas flows to the center of NGC 1672. Dust streamers plunge from near the end of the bar into the bar itself, and then directly to the center. This direct path means that gas inflow is rapid. A comparison of the inflow rate to the gas reservoir gives the timescale for accretion. In a study of NGC 1365 (Elmegreen et al. 2009c), which looks like NGC 1672, the timescale for gas exhaustion is only $\sim 0.5$ Gyr. If the initial reservoir of gas was comparable to what is there today, then this short time is also comparable to the age of the bar. That is, bars like these have to be fairly young, $\sim 1$ Gyr, or the gas would have been cleared out by now.

NGC 1365 and many other barred galaxies have massive clusters in their ILR regions - much more massive than typical disk clusters or clusters forming in spiral density wave arms. Perhaps this difference is only a size-of-sample effect if the ILR regions of bars have higher overall star formation rates. Or, star formation in bar ILRs may differ from that in main disks. 


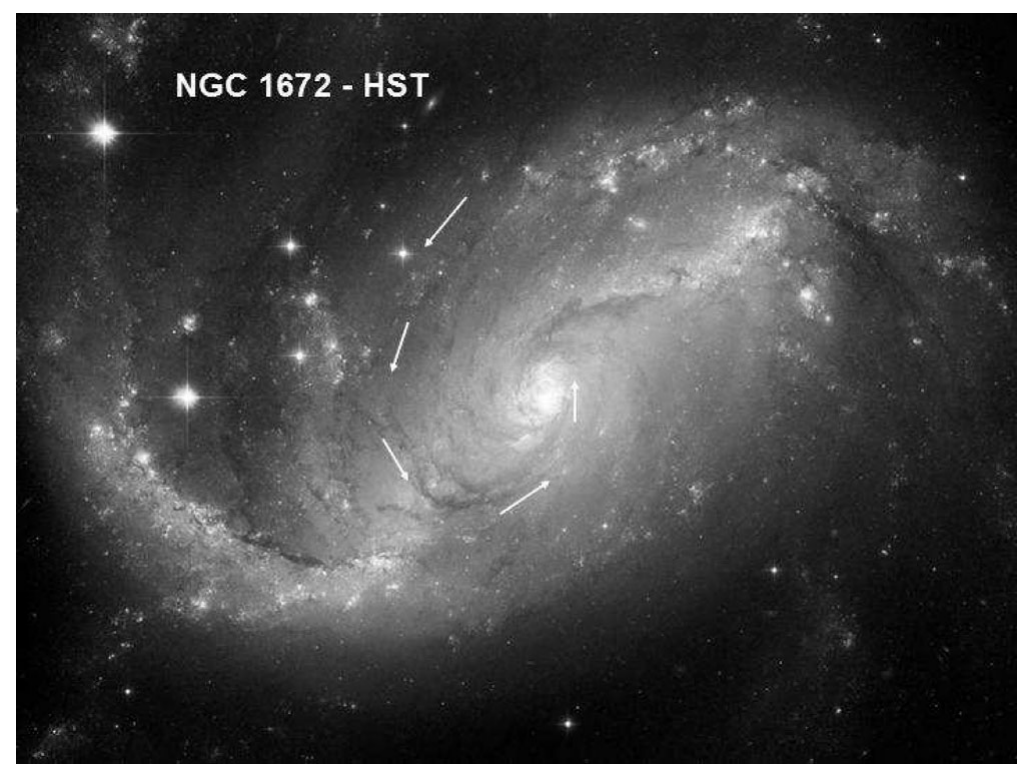

Figure 4. Barred galaxy NGC 1672 from the Hubble Heritage Team. Dust lanes suggest the gas flow pattern toward the center (arrows), where star formation is very active.

\section{Super-Bubbles, Super-Rings, and Colliding Flows}

Figure 1 shows a spiral arm segment with large rings of gas and star formation downstream from the arms. These are giant cavities are made by star formation, as discussed above. The solar neighborhood is downstream from the Carina spiral arm and also has a large cavity apparently made by star formation. This is associated with the expanding HI ring discovered by Lindblad et al. (1973) with a hole in the diffuse dust (Lallement et al. 2003). The size of the dust hole is $100 \mathrm{pc}$ by $300 \mathrm{pc}$, and it extends all the way through the disk. The cavities in Figure 1 are about the same size, and larger than the ISM thickness. Thus they are rings rather than shells. The smaller regions could be three-dimensional shells.

Expanding rings are more unstable than expanding shells because the self-gravitational force vectors that drive gas collection in a one-dimensional section of a ring are all directed toward the growing condensation. According to Elmegreen (1994), the time for significant collapse in an expanding shell is $\sim 100(n \mathcal{M})^{-0.5}$ Myr for ambient density $n$ in $\mathrm{cm}^{-3}$ and Mach number $\mathcal{M}$, and the time in an expanding ring is $124\left(n \mathcal{M}^{2}\right)^{-0.5}$ Myr. The ring expression contains a stronger dependence on the Mach number, and this give the ring a shorter collapse time.

Generally, the expansion scale equals the shock speed multiplied by the time, and the shock speed is about $\left(P / \rho_{0}\right)^{0.5}$ for driving pressure $P$ and preshock density $\rho_{0}$. If the relevant time is the collapse time, $\left(G \rho_{\text {comp }}\right)^{-0.5}$ for compressed density $\rho_{\text {comp }}$, then the expansion scale is, after rearrangement, $\left(P / \rho_{\text {comp }}\right)^{0.5}\left(G \rho_{0}\right)^{-0.5}$, which is the velocity dispersion in the compressed region multiplied by the dynamical time in the ambient gas. Details about the compression source drop out.

If the expansion scale is less than the cloud scale, then pillars and bright rims form by the push-back of interclump gas (Elmegreen, Kimura \& Tosa 1995, Gritschneder et al. 2009). Star formation is a fast process (squeezing pre-existing clumps), the velocity of triggered stars is small, and causality is difficult to determine, i.e., stars could have formed in the clumps anyway. If the expansion scale is larger than the cloud scale, then shells 
and rings form by the push-back of all ISM gas (e.g. Dale et al. 2011), triggering is a slow process because new clumps have to form on a timescale of $\left(G \rho_{\text {shell }}\right)^{-0.5}$, and the velocity of triggered stars can be large, on the order of the shock speed, $\left(P / \rho_{0}\right)^{1 / 2}$. There is also a clear causality condition because two stellar generations have to be separated by a distance equal to the age times the mean velocity.

A popular cloud formation scenario considers the compression of gas between two "colliding flows" (e.g., Heitsch et al. 2008, Audit \& Hennebelle 2010). Shell or ring accumulation forms clouds too, but is different in several ways. Shells and rings have a lateral expansion as they expand radially, and this lateral expansion resists gravitational collapse. Shells and rings also decelerate so that newly formed condensations protrude out the front and have the potential to erode. Shells and rings have a shock on only one side. A shock boundary condition causes a diverging flow at each clump, and this divergence resists collapse. The pressure boundary condition on the other side of the shell or ring squeezes the perturbations and aids collapse. For colliding flows with constant velocity streams, there is a shock on each side and no acceleration of the condensation between them when it is in equilibrium. We observe shells and rings commonly, as shown in the figures and in surveys (e.g., Könyves et al. 2007, Ehlerová \& Palouš 2005), but there is no clear evidence yet for cloud formation on GMC-scales by colliding flows (on much larger scales, the collision between two galaxies can have a colliding flow; Herrara et al. 2011). Still, colliding flows are a good model to study molecule formation and fragmentation in a dynamic environment.

Triggering in the ring RCW 79 was studied in detail by Zavagno et al. (2006), who suggested there was a collapsed neutral region containing young stars, 0.1 Myr old, along the periphery of a swept-up region that was $1.7 \mathrm{Myr}$ old. There are several neutral condensations in this shell, somewhat equally spaced around part of the projected edge. Deharveng et al. (2010) studied 102 Milky Way bubbles and suggested that 18 of them have either ultracompact HII regions or methanol masers along their edge, suggesting triggering of massive stars in swept-up gas.

\section{Bright Rims and Pillars}

Most regions of massive star formation have bright ionized rims and pillars of neutral gas pointing to the sources of radiation. A well-studied example is IC 1396, which is a circular HII region $12 \mathrm{pc}$ in radius, with an expansion speed of $5 \mathrm{~km} \mathrm{~s}^{-1}$ and an age of 2.5 Myr (Patel et al. 1995). On the western edge is a neutral pillar several pc long (Fig. 5 ) with very young (class I - diamonds) stars in head-like protrusions and other young stars (class II - circles) all around (Reach et al. 2009). This is a typical case.

There is often an age gradient in pillars with the oldest stars closer to the center of the HII region (Sugitani et al. 1995). The timescale for triggering can be very fast, $10^{4}$ yrs (Sugitani et al. 1989), but the age gradient can span a time of $10^{6}$ years or longer during which the compression moves down the pillar (Smith 2010).

This mechanism of triggering was originally illustrated in models by Klein et al. (1983). Recent simulations by Bisbas et al. (2011) compare radiative implosion in a variety of conditions. At low incident flux, the implosion is slow, the bright rim is wide, and star formation is far from the tip in the center of a converging compression front. At high incident flux, the implosion is fast, the pillar is narrow, and star formation is close to the tip where the initial compression was concentrated. At late times, the pillar can be long and thin, with bare stars near the head and other stars embedded throughout. If there are many small clumps in the original cloud, then there can be many small pillars, one for each clump, with star formation in each. 


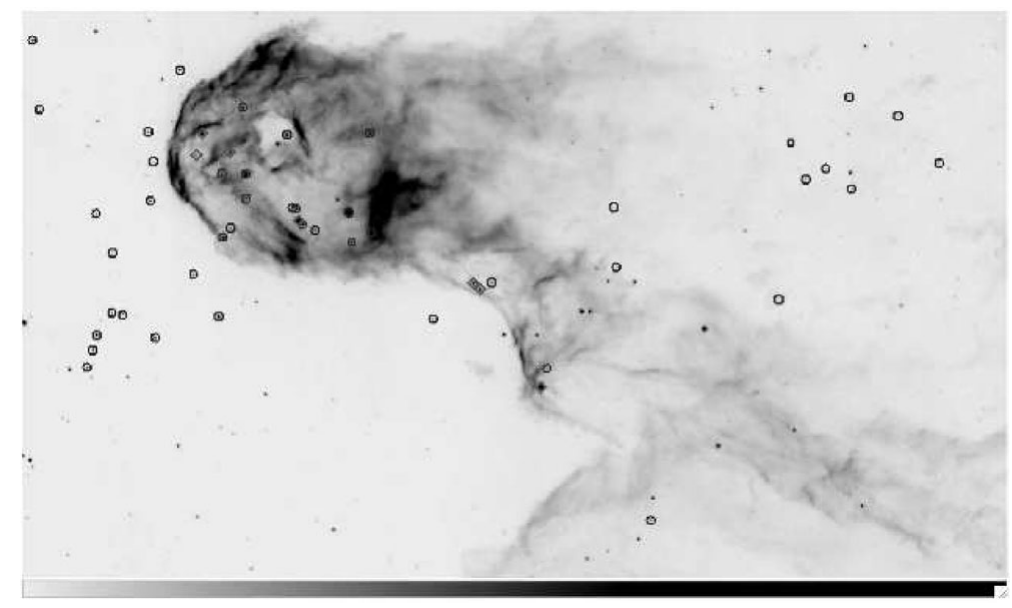

Figure 5. Bright rim at the edge of the HII region IC 1396, from Reach et al. (2009). The youngest stars are denoted by diamonds. They were probably triggered to form in compressed cloud clumps that were exposed by ionization and movement of the lowest density, surrounding regions.

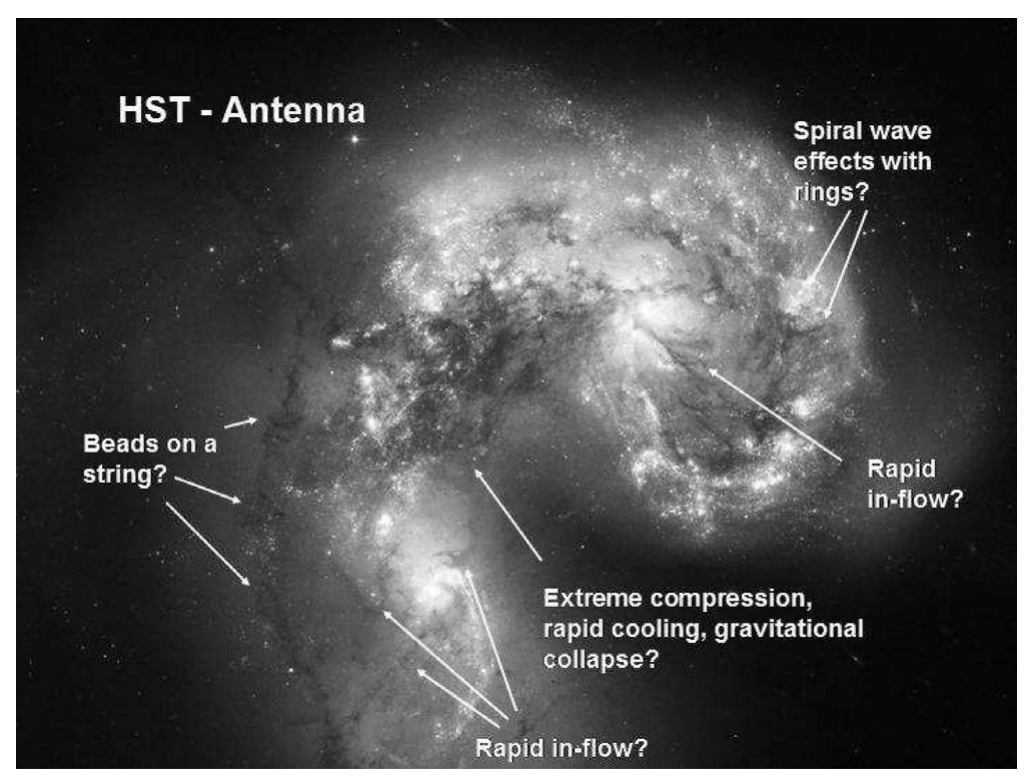

Figure 6. HST image of the Antenna galaxy showing several types of triggering discussed in the text.

\section{The Antenna Galaxy: a Mixture of Triggering Processes}

Several of the triggering mechanisms discussed above are illustrated in the Antenna galaxy, which is a merger. Figure 6 shows the Antenna labeled with suggested analogies: spiral arm shock triggering with downstream rings, beads on a string from gravitational collapse in a filament, radial inflow in elongated, bar-like regions, and a converging flow that produces extreme compressions between the two gas disks. 


\section{Triggering and Empirical Star Formation Laws}

Star formation triggering is about when, where, and how star formation begins. The global laws of star formation, such as the Kennicutt (1998) or Bigiel et al. (2008) relations, show a correlation between the star formation rate and the supply of gas of various types. The Kennicutt (1998) study shows a star formation rate per unit area that scales with the 1.4 power of the total gas column density in the main disks and central regions of spiral galaxies, and the Bigiel et al. (2008) and Leroy et al. (2008) studies show a linear relation between the star formation rate per unit area and the $\mathrm{CO}$ emission per unit area. A linear relation for dense gas traced by HCN was first shown by Gao \& Solomon (2004) and Wu et al. (2005).

In all cases, star formation is assumed to occur only in cold dark gas, which is traced by $\mathrm{CO}$ at low density and $\mathrm{HCN}$ and other molecules at high density. The empirical laws suggest that the star formation rate is independent of how the gas is assembled, and therefore independent of the triggering mechanisms. This is particularly true if the average pressure and radiation field determine the general molecular state of the gas, and the pressures which make shells and rings do not change this state much, they just push the gas around. Triggering will also not influence the empirical laws much if the various types of triggering are always present to the same degree. For example, if every initial incidence of star formation is followed by a certain proportion of additional stars that form by radiative implosion or in shell and ring collapse, then the conversion efficiency from ambient gas to young stars will contain all of these effects combined. Each triggering process will not stand out separately in the total star formation rate.

According to Gnedin \& Kravtsov (2011) and others, CO and star formation are predictable from the average pressure, self-shielding, radiation field, and other quantities. The pressure that determines molecular self-shielding does not vary much on the scale of shells and rings once they are old enough for star formation to begin. Regions with radiative implosion have high pressure, but this may be a relatively minor star formation event. The pressure varies in spiral arms, but this should only put scatter in the empirical relations. Thus the signatures of localized triggering may be imperceptible in the empirical star formation laws.

\section{All you need is cold gas: the legacy of K.E. Edgeworth}

The universality of the empirical laws combined with the evidence for star formation in shells, spiral arms, pillars, and other pressurized structures suggests that the primary ingredient for star formation is cold gas and not a particular geometry. Cold gas means colder than thermal equilibrium commonly gives for atomic gas in the background galactic radiation field. Cold gas for star formation implies shielding from starlight and the presence of molecules for rotational cooling, which can operate down to a few degrees above the microwave background. Once the gas is shielded from starlight and turns molecular, background pressure and turbulent motion will compress it to a high density in small regions. Then gravity becomes important and the thermal Jeans mass drops to be comparable to the region mass. Star formation usually follows.

Shell, ring, or pillar-like triggering and spiral waves might be more important as a net positive contributor to star formation in regions where the molecular fraction is moderately low on average, i.e., at the boundary between highly molecular inner disks of galaxies and highly atomic outer disks. In the inner disks, the gas pretty much stays molecular everywhere because of the high pressure from its weight in the disk, and because of the high opacity. Starlight ionizes and photodissociates molecules at the cloud 
edges (Heiner et al. 2011) and between the clouds. Cold gas is present in most clouds, so additional pressures from young stars or spiral waves will not make the gas much colder or more molecular. In the far-outer regions, however, it is rather difficult to turn atomic gas into molecular gas because the average pressure and opacity are very low. Then superbubbles might not trigger new star formation, but only make diffuse atomic shells which have little cold gas. Between these two zones, or perhaps in dwarf irregular galaxies where the same intermediate conditions apply, triggering by the pressures of young stars might make more of a difference in the total star formation rate.

An observational test of this prediction would be to measure the radial variation of the ratio of the normalized star formation efficiency in compressed and non-compressed regions. The normalized efficiency is the star formation rate per unit gas mass per unit dynamical rate. An example would be the ratio of the efficiency in the spiral arms to the interarm regions. If this ratio increases with radius, then spiral arm triggering is doing more to enhance star formation in the outer parts of galaxies, where the gas is mostly atomic, than in the inner parts, where the gas is mostly molecular. Alternatively, one could plot this ratio versus the average molecular fraction instead of the radius.

The overriding importance of cold gas for star formation was originally recognized by Kenneth Essex Edgeworth in 1946 - long before the modern star formation era. (For a biography of Edgeworth, see Hollis 1996.) At that time, most stars, like the Earth and the whole universe, were thought to be about 3 Gyr old, star formation was not considered to happen in the present day, except possibly for the most massive stars (which were known to burn their fuel quickly), and the stability of galactic disks was not yet understood. Edgeworth and others were thinking about star formation mostly in the context of the beginning of the universe. He noted from a thermal Jeans analysis that star formation by gravitational instability in a galaxy disk, using an equation like eq. 1.1, requires a temperature of $\sim 2.8 \mathrm{~K}$, which he considered "very improbable." He also said that angular momentum from galactic rotation was too large for the solar system to form, and suggested that stars might form instead by successive condensations to overcome the angular momentum problem. This led him to "expect to find that the majority of the stars were members of star clusters" which is "not in agreement with observation." Finally, he suggested that the rotating gas disk of a galaxy, at $\sim 1000 \mathrm{~K}$, breaks up into azimuthal filaments, which then break up into stars following the removal of heat. He went on to suggest that the residual material around each star makes planets.

These ideas are all essentially correct by modern standards - even though Edgeworth did not believe or observe them at the time. Edgeworth understood that star formation requires very cold gas, it most likely occurs in massive aggregates, and it should be patterned as beads on a string for the galactic scale. These ideas were too far ahead of their time to have much influence. Star formation as we know it was discovered several years later when Ambartsumian (1949) showed that local OB associations are expanding away from a common center. This limited their age to $10 \mathrm{Myr}$.

\section{Summary}

A variety of processes cause interstellar gas to become cold enough and dense enough to form stars. On galactic scales, stellar instabilities, spiral waves, and global perturbations like bars can move the gas around supersonically and cause shocks to form that are larger than the characteristic size of a gravitational instability in the gas. Then giant cloud complexes form from the ambient gas. As these complexes dissipate their internal turbulent energy, they contract gravitationally and fragment because of converging and diverging turbulent motions until dense, thermally-dominated cold cloud cores form. 
Stellar pressures also compress the gas supersonically. On sufficiently large scales, these compressions lead to collapse in shells and rings. On small scales, stellar pressures can turn in pre-existing clumps unstable to collapse, especially along the edges of HII regions and super-bubbles.

The empirical laws of star formation have no obvious connection to the details of these triggering mechanisms. The empirical laws state mostly that star formation requires cold and dense gas. In the case of the Kennicutt (1998) law, with its non-linear dependence of star formation rate on total gas density, empirical evidence suggests also that general dynamical processes in the ISM are involved in determining the time scale. Since triggering on the length scale of these laws has the same dynamical time scale, all of the various triggering processes can mix together without much distinction.

The distinct contribution that triggering makes to the star formation rate might be most evident in large-scale regions where the average molecular fraction is neither very high nor very low. There the dynamical processes related to cloud formation could have a significant influence on the abundance of cold gas in clouds. There might still be a linear relation between cold gas mass and star formation rate at these places, because star formation follows cold gas no matter what forms the cold gas, but the rate of both cold gas formation and star formation could be modulated by dynamical processes more there than elsewhere.

The author is grateful to the National Science Foundation for support from grant AST0707426, and to the conference organizers, particularly Professors Cristina Popescu and Richard Tuffs, for their support and hospitality.

\section{References}

Ambartsumian, V. A. 1949, Soviet AJ, 26, 3

Athanassoula, E. 1992, MNRAS, 259, 345

Audit, E. \& Hennebelle, P. 2010, A\& $A, 511,76$

Bigiel, F., Leroy, A., Walter, F., et al. 2008, AJ, 136, 2846

Bisbas, T. G., Wünsch, R., Whitworth, A. P., Hubber, D. A., \& Walch, S. 2011, ApJ, 736, 142

Daddi, E. et al. 2010a, ApJ, 713, 686

Dale, D. A. et al. 2009, ApJ, 703, 517

Dale, J. E., Wünsch, R., Smith, R. J., Whitworth, A., \& Palouš, J. 2011, MNRAS, 411, 2230

Davies, R., Förster Schreiber, N. M., Cresci, G., et al. 2011, ApJ, 741, 69

Deharveng, L., Schuller, F., Anderson, L. D., et al. 2010, A\& A, 523, 6

Desai, K. M., Chu, Y.-H., et al. 2010, AJ, 140, 584

Edgeworth, K. E. 1946, MNRAS, 106, 470

Efremov, Yu. N. 2010, MNRAS, 405, 1531

Ehlerová, S. \& Palouš, J. 2005, A\&A, 437, 101

Elmegreen, B. G. 1987, ApJ, 312, 626

Elmegreen, B. G. 1994, ApJ, 427, 384

Elmegreen, B. C. 2011, ApJ, 737, 10

Elmegreen, B. G., Kimura, T., \& Tosa, M. 1995, ApJ, 451, 675

Elmegreen, B. G., Elmegreen, D. M., Fernandez, M. X., \& Lemonias, J. J. 2009a, ApJ, 692, 12

Elmegreen, B. G., Galliano, E., \& Alloin, D. 2009, ApJ, 703, 1297

Elmegreen, D. M. 1980, ApJS, 43, 37

Elmegreen, D. M. \& Elmegreen, B. G. 1995, ApJ, 445, 591

Elmegreen, D. M., Elmegreen, B. G., Marcus, M., et al. 2009b, ApJ, 701, 306

Elmegreen, D. M., Elmegreen, B. G., Yau, A. et al. 2011, ApJ, 737, 32

Gao, Y. \& Solomon, P. M. 2004, ApJ, 609, 271

Genzel, R., Newman, S., Jones, T., et al. 2011, ApJ , 733, 101 
Gil de Paz, A. et al. 2007, ApJS, 173, 185

Gnedin, N. Y. \& Kravtsov, A. V. 2011, ApJ, 728, 88

Grabelsky, D. A., Cohen, R. S., Bronfman, L., Thaddeus, P., \& May, J. 1987, ApJ, 315, 122

Gritschneder, M., Naab, T., Walch, S., Burkert, A., \& Heitsch, F. 2009, ApJ, 694, L26

Heiner, J. S., Allen, R. J., \& van der Kruit, P. C. 2011, MNRAS, 416, 2

Heitsch, F., Hartmann, L. W., \& Burkert, A. 2008, ApJ, 683, 786

Herrera, C. N., Boulanger, F., \& Nesvadba, N. P. H. 2011, A\&AA, 534, 138

Hollis, A. J. 1996, J. British Astron. Assoc., 106, 354

Jog, C. J. \& Solomon, P. M. 1984, ApJ, 276, 114

Julian, W. H. \& Toomre, A. 1966, ApJ, 146, 810

Kennicutt, R. C., Jr. 1998, ApJ, 498, 541

Kim, W.-T. \& Ostriker, E. C. 2002, ApJ, 570, 132

Kim, W.-T., Ostriker, E. C., \& Stone, J. M. 2002, ApJ, 581, 1080

Klein, R. I., Sandford, M. T., \& II, Whitaker, R. W. 1983, ApJL, 271, 69

Könyves, V., Kiss, Cs., Moór, A., Kiss, Z. T., \& Tóth, L. V. 2007, A\&̊A, 463, 1227

Lallement, R., Welsh, B. Y., Vergely, J. L., Crifo, F., \& Sfeir, D. 2003, A\&\&A, 411, 447

Leroy, A. K., Walter, F., Brinks, E. et al. 2008, AJ, 136, 2782

Lin, C. C. \& Shu, F. H. 1964, ApJ, 140, 646

Lindblad, P. O., Grape, K., Sandqvist, A., \& Schober, J. 1973, A\&A, 24, 309

Matsuda, T. \& Nelson, A. H. 1977, Nature, 266, 607

Patel, N. A., Goldsmith, P. F., Snell, R. L., Hezel, T., \& Xie, T. 1995, ApJ, 447, 721

Rafikov, R. R. 2001, MNRAS, 323, 445

Reach, W. T., et al. 2009, ApJ, 690, 683

Roberts, W. W. 1969, ApJ, 158, 123

Romeo, A. B. \& Wiegert, J. 2011, MNRAS, 416, 1191

Smith, N. 2010, MNRAS, 406, 952

Sugitani, K., Fukui, Y., Mizuni, A., \& Ohashi, N. 1989, ApJL, 342, 87

Sugitani, K., Tamura, M., \& Ogura, K. 1995, ApJL, 455, 39

Tacconi, L., et al. 2010, Nature, 463, 781

Toomre, A. 1969, ApJ, 158, 899

Toomre, A. 1981, in: S.M. Fall (ed.), The structure and evolution of normal galaxies, (Cambridge: Cambridge University Press), p. 111.

Wu, J., Evans, N. J., I. I., \& Gao, Y., et al. 2005, ApJ, 635, L173

Zavagno, A., Deharveng, L., Comerón, F., et al. 2006, A\&گA, 446, 171

\section{Discussion}

GAO: You mentioned that supernovae are not important in triggering star formation on galactic scales. Could they be important in extreme starbursts where essentially most of the molecular gas concentrated within a few hundred parsec, experiencing constant impacts of supernovae due to the several orders of magnitude higher star formation ratee?

Elmegreen: There is no observational answer to this question now, but a guess based on theory is that supernova remnants in high density environments are very short-lived because the radiation rate is high. If their lifetime is less than the dynamical time of the surrounding medium, then triggering gets different. The prior HII region of that supernova star might have had a bigger triggering effect, if it was a massive star.

TufFs: We know that more massive galaxies form stars more efficiently (out of available gas) than low mass galaxies. Can you comment on how the small scale processes you have described relate to global galaxian properties? For example, does the expected differing vertical scale of gas in different gravitational potentials affect the confinement of the ring structures you described? 
Elmegreen: The ISM Jeans length determines both the thickness of a disk and the size of its largest star forming regions. So relatively thick gas disks, as in late type galaxies, have relatively bigger star complexes. Their blow out from shells and rings should be about the same as in relatively thin gas disks. The reason why massive galaxies form stars with a higher specific star formation rate during their most active period is probably related to the average density and average intergalactic accretion rate during this period in their evolution. 